\title{
Optomechanics with silicon nanowires by harnessing confined electromagnetic modes
}

\author{
Daniel Ramos ${ }^{\dagger, \|}$, Eduardo Gil-Santos ${ }^{\ddagger, \|}$, Valerio Pini ${ }^{\ddagger}$, Jose M. Llorens ${ }^{\ddagger}$, Marta \\ Fernández-Regúlez ${ }^{\S}$, Álvaro San Paulo ${ }^{\S}$, M. Calleja ${ }^{\ddagger}$ and J.Tamayo*,‡ \\ ${ }^{\dagger}$ School of Engineering and Applied Sciences Harvard University, Cambridge, MA, 02138, USA \\ Instituto de Microelectrónica de Madrid, CSIC. Isaac Newton 8 (PTM) Tres Cantos 28760 Madrid. Spain \\ ${ }^{\S}$ Instituto de Microelectrónica de Barcelona, CSIC. Campus UAB, Bellaterra 08193, Barcelona, Spain.
}

S Supporting Information

ABSTRACT: The optomechanical coupling that emerges in an optical cavity in which one of the mirrors is a mechanical resonator has allowed sub-Kelvin cooling with the prospect of observing quantum phenomena, and self-sustained oscillators with very high spectral purity. Both applications clearly benefit from the use of the smallest possible mechanical resonator. Unfortunately, the optomechanical coupling largely decays when the size of the mechanical system is below the light wavelength. Here, we propose to exploit the optical resonances associated to the light confinement in subwavelength structures to circumvent this limitation, efficiently extending optomechanics to nanoscale objects. We demonstrate this mechanism with suspended silicon nanowires. We are able to optically cool the mechanical vibration of the nanowires from room temperature to $30-40 \mathrm{~K}$ or to obtain regenerative mechanical oscillation with a frequency stability of about one part per million. The reported optomechanical phenomena can be exploited for developing cost-optimized mass sensors with sensitivities in the zeptogram range.

KEYWORDS: optomechanics, nanomechanical sensors, silicon nanowires, NEMS, force detection, mass sensing 
Micromechanical resonators such as microcantilevers have become the basis of a wide variety of applications from scanning probe microscopy ${ }^{1}$ to mass sensing ${ }^{2-5}$. The concept is the measurement of the resonance frequency variations; that in the case of scanning probe microscopy arise from the intermolecular forces between a sharp tip attached to a microcantilever and the probed surface, and in the case of mass sensing are induced by the mass added on the surface of the resonator. The recent efforts for reducing the size of the resonators to the nanoscale has allowed the attainment of unprecedented sensitivities of zeptogram-scale mass sensing ${ }^{6-12}$ and zeptonewton-scale force detection ${ }^{13-14}$. These achievements have enabled milestones such as single electron-spin paramagnetic resonance ${ }^{13}$ and protein spectrometry ${ }^{11}$. In parallel, the optomechanics field has rapidly grown for exploiting the forces exerted by the electromagnetic radiation on mechanical structures ${ }^{15-22}$. A common experimental set-up consists of positioning a freestanding mechanical element with a high reflectivity coating near a mirror to establish a Fabry-Pérot cavity with high finesse. More complex embodiments include coupling the mechanical element to microtoroidal cavities ${ }^{19}{ }^{23}$, to a high-finesse Fabry-Pérot cavities formed between two macroscopic rigid mirrors ${ }^{24}$ and the use of external phononic bandgap shields ${ }^{25}$. A primary goal of optomechanics is to exploit the optical forces that emerge in the cavity to cool down the fundamental vibration mode of the mechanical system. This goal has inspired analogue methodologies such as the electromechanical coupling between a mechanical resonator and a quantum bit $^{26-27}$. We believe that the advances in optomechanics can also be exploited for enhancing the detection limits of nanomechanical sensors. However, when the mechanical system dimensions are shrunk beyond the optical wavelength, strong diffraction effects emerge and the optical back-action is largely reduced ${ }^{20}$. Here, we find that silicon nanowires support optical resonances associated to the light confinement within the nanowire that can be harnessed to achieve an unprecedented optomechanical coupling in these tiny structures. This is the first demonstration of optomechanics with nanoscale mechanical resonators.

The measurements presented in this work were performed on silicon nanowires grown horizontally by the vapour-solid-liquid mechanism at the sidewalls of prepatterned microtrenches (Fig. 1a). The fabrication is described in the supplementary material. The resulting singly clamped nanowire beams have a length of 6-8 $\mu \mathrm{m}$ and diameters ranging from 100 to 200 nanometers. The distance between the nanowire and the substrate lies in the 1100 $1700 \mathrm{~nm}$ range. Each nanowire and the substrate underneath form an optical nanoscale cavity that allows the measurement of the Brownian motion of the nanowire with picometer accuracy by laser interferometry ${ }^{28-31}$ (fig. 1b). The measurements were carried out in high vacuum $\left(10^{-7}\right.$ Torr) at room temperature. In order to amplify the laser-back action, we deposited an $8 \mathrm{~nm}$ thick gold layer on top of the nanowire ${ }^{32}$. The purpose of the thin gold layer is to transform the 
light energy into a mechanical force via an intermediate thermal stage ${ }^{33}$. This force is usually referred to as photothermal force. Since the Au-coating breaks the cross-section symmetry of the nanowire, the nanowire vibrates into two orthogonal directions, parallel (not detectable) and perpendicular to the substrate at significantly different frequencies ${ }^{28}$. Hereinafter we will focus on the orthogonal vibration mode.

The nanowires used in this work have an effective mass of 50-80 fg that is more than two orders of magnitude smaller than the lightest mechanical mirror previously used in cavity optomechanics ${ }^{20}$. In fact, resonant silicon nanowires are promising candidates for mass sensors on the verge of single atom resolution and for single protein spectrometry ${ }^{9,28}$. Our theoretical calculation indicate that the cavity finesse that describes the number of reflections a photon undergoes before escaping the cavity formed by the nanowire and the substrate underneath is very small $\sim 1$ (see supplementary material). This is a direct consequence of the light scattering produced by the subwavelength hexagonal cross-section of the nanowire (see supplementary material). This raises the question of how the nanomechanical response of the nanowire can efficiently be coupled to the light field. It has recently been demonstrated that there exist optical resonances that confine the light in subwalength and high-refractive-index semiconductor nanostructures ${ }^{34-35}$. Here, we have calculated the optical absorption of a gold-coated nanowire free in space as a function of the diameter at a wavelength of $633 \mathrm{~nm}$ (fig. 1c). The theoretical curve shows a series of optical resonances in which the nanowires strongly absorb the light. The spectrum can be associated, to a very good approximation, with the morphology-dependent optical resonances (also known as whispery gallery modes or just Mie resonances). These resonances can be viewed as light trapped in circulating orbits by multiple internal reflections. The electric field distribution of these optical resonances is shown in the insets of fig. 1c. For the selected diameters and wavelengths, three optical resonances within the nanowire can be excited that correspond with the modes $(1,0),(2,0)$ and $(0,1)$, where the first index is the azimuthal mode number that indicates the effective number of wavelengths around the nanowire circumference and the second index is the radial mode number that describes the number of radial field nodes within the nanowire. Owing to the small size of the nanowire, the resonant modes are leaky and are able to interact with the near environment. Given the poor quality of the optical cavity, our system is more conveniently described in terms of the interaction between the nanowire and the light standing wave that arises from the interference between the incoming and reflected off the substrate laser beams. The question now is if we are able to couple the nanowire-confined optical modes to the interference standing wave in order to achieve an efficient optomechanical system based on a nanowire. 
Figure $2 \mathrm{a}$ shows the frequency spectra of the fundamental vibration mode extracted from the Brownian fluctuations for two different nanowires, referred to as NW 1 and NW 2. The measurements were performed at two wavelengths, $543 \mathrm{~nm}$ and $633 \mathrm{~nm}$ with a laser power of $500 \mu \mathrm{W}$ (a schematic of the experimental set-up is shown in supplementary materials). Both nanowires are separated from the substrate by $1450 \mathrm{~nm}$, however the diameters of NW 1 and NW 2 are 150 and $180 \mathrm{~nm}$, respectively. With the selected diameters and wavelengths, we expect to excite the $(1,0)$ and $(2,0)$ optical resonances. We choose the same distance to the substrate to remark that the optomechanical phenomena observed with nanowires cannot be explained in terms of a Fabry-Perot cavity, in which the optomechanical coupling is solely controlled by external cavity detuning. When the red laser was used, NW 1 exhibited a quality factor of 173000 and an effective temperature of $20000 \mathrm{~K}$; whereas NW 2 showed a quality factor of 320 and an effective temperature of 30-40 K. The quality factor of both nanowires was 3000-3500 when the laser intensity was minimized. By changing the laser "color" from red to green, we modified the spatial period of the interference standing wave giving rise to a switch in the response of nanowires NW1 and NW2. The vibration of NW 1 was cooled down to an effective temperature of $100 \mathrm{~K}$ and the quality factor was reduced to 1000 , whereas the vibration of NW 2 was amplified with an effective quality factor and temperature of 65000 and $6100 \mathrm{~K}$, respectively.

We show in figure $2 b$ the amplitude at resonance as a function of the laser power at 633 $\mathrm{nm}$ for two nanowires separated from the substrate $1520 \mathrm{~nm}$, with diameters of $120 \mathrm{~nm}$ and 180 nm. The thinner nanowire exhibits optical cooling whereas the other shows optical amplification. In the case of cooling, the amplitude decreases about six times by increasing the laser power from 50 to $500 \mu \mathrm{W}$. The achieved values of the effective quality factor and temperature are of 600 and $50 \mathrm{~K}$, respectively. In the case of the optical amplification, for a laser power of $350 \mu \mathrm{W}$, the quality factor is of 263000 that corresponds to an extremely small linewidth of $23 \mathrm{~Hz}$. For the sake of a major understanding, we model the system as a closed loop consisting of two elements, the mechanical resonator that translates incoming forces into vibration and the electromagnetic field that translates the vibration into a photothermal force (fig. 2c). Since the feedback loop is passive, the thermal Langevin force and optical readout elements stay out of the closed-loop. A delay comes up between the feedback force and the resonator vibration due to the heat diffusion time. Only the out-of-phase component of the feedback force can coherently amplify or cool down the motion of the mechanical resonator as it directly modifies the effective damping rate of the system. The feedback loop can be approximately described by means of the harmonic oscillator differential equation ${ }^{36}$ : $m \ddot{z}(t)+\gamma \dot{z}(t)+k z(t)=F_{t h}(t)+g \dot{z}(t) / \omega_{0}$, where $z$ represents the nanowire tip motion, $m$ is the effective mass, $\gamma$ is the damping coefficient, $k$ is the spring constant and $g$ is the 
optomechanical feedback gain that must be proportional to the light intensity. The optomechanical feedback force modifies the damping coefficient to $\gamma_{\text {eff }}=\gamma-g / \omega_{0}$ and consequently the effective temperature to $T_{\text {eff }}=\left(\frac{y}{\gamma_{\text {eff }}}\right) T$ in such a way that the fluctuationdissipation theorem is satisfied. The amplitude at resonance for a measurement bandwidth $\Delta \omega$ is then given by,

$$
A_{0}=2 \frac{\sqrt{\gamma k_{B} T \Delta \omega}}{\omega_{0}\left(\gamma-g / \omega_{0}\right)}
$$

The experimental data (symbols in fig. $2 \mathrm{~b}$ ) are fitted to equation (1) giving rise to an optomechanical feedback gain $(g)$ per laser power unit in the range of $0.1-1 \mathrm{pN} / \mathrm{nm}$ per $\mathrm{mW}$.

These experimental results are consistent with our hypothesis that the confined optical resonances supported by the nanowire can be used to develop an efficient optomechanical system. To obtain further demonstration of this concept, we performed numerical calculations to obtain the optomechanical feedback gain. The calculations show that the radiation pressure can be neglected as the photon lifetime in the nanocavity is much smaller than the oscillation period of the nanowire and the resulting force is small, of few tens of $\mathrm{fN}$. The model is detailed in the supplemental material. Briefly, after solving the Maxwell equations, we solve the heat diffusion equation for obtaining the temperature variation induced by the mechanical fluctuation of the nanowire in the interference field. Finally, we apply the Euler-Bernoulli beam theory for obtaining the out-of-phase photothermal force that the nanowire experiences when it moves off its equilibrium position. Figure $3 \mathrm{a}$ shows the colour intensity maps of the calculated optomechanical feedback gain per unit of light power as a function of the nanowire diameter and the distance to the substrate, both normalized to the wavelength of $633 \mathrm{~nm}$. A positive value of the gain leads to the amplification of the fundamental vibration mode, whereas a negative value leads to its cooling. In addition, we have plotted the experimental values of diameter and distance coordinates of the nanowires that exhibited optical amplification (red circles) and cooling (cyan circles). The distance and the diameters were measured by scanning electron and atomic force microscopies. We find a very good agreement between the experimental data and the proposed theoretical model. In addition, the calculated values of the optomechanical feedback gain are in consistency with the experimental values derived from fig. $2 b$. This finding strongly suggests that when the nanowires are near a substrate, the leaky resonant modes excited within the nanowire act as an "antenna" that selectively amplifies the interference standing waves formed in the nanocavity. This coupling means that the optical resonant modes can be amplified or suppressed by modifying the distance between the nanowire and the substrate. The amplification or extinction distance depends on the electric field configuration of 
the optical resonance, as illustrated in figure $3 \mathrm{~b}$ where we plot the absorption for the resonant modes $(1,0)$ and $(2,0)$ at distances of 1250 and $1400 \mathrm{~nm}$, respectively. The electric field distributions of these optical resonances at both separations are shown in fig. 3c.

Finally, we apply the discovered optomechanical phenomena to induce self-sustained oscillations in nanowires with very high spectral purity. This state is reached when the gain of the feedback loop reaches a critical value in which the damping rate becomes negative. The transient oscillation then grows exponentially until the non-linear saturation mechanisms of the system come into play and the oscillation reaches a steady state with virtually no noise in the amplitude and small phase fluctuations of diffusive nature ${ }^{36-38}$. Here the saturation comes from the non-linear behaviour of the light intensity modulation. The transition from optical amplification to self-oscillation is seen as an abrupt increase of the amplitude of several orders of magnitude when the laser intensity reaches a critical value that is of $310 \mu \mathrm{W}$ in the case shown in figure $2 \mathrm{~b}$. In figure $4 \mathrm{a}$, we characterize the frequency stability of the self-sustained oscillation of the nanowire for a laser power of $350 \mu \mathrm{W}$. The frequency fluctuations for an averaging time of $1 \mathrm{~s}$ were of $3 \times 10^{-6}$ during one hour of experiment. Figure $4 \mathrm{~b}$ shows the standard deviation of the frequency as a function of the integration time. For integration times of about one minute the frequency fluctuations are reduced to $10^{-7}$. The ultimate limit in the frequency noise of the self-sustained oscillation is imposed by the phase diffusion that approximately follows the Einstein equation for diffusion processes ${ }^{36,38}$. The corresponding frequency noise is given by $\frac{\delta \omega_{0}}{\omega_{0}} \approx 0.341\left(\frac{k_{B} T}{Q k A_{s}{ }^{2} \omega_{0} \tau}\right)^{\frac{1}{2}}$, where $\mathrm{k}_{\mathrm{B}}$ is the Boltzmann constant, $\mathrm{T}$ is the temperature, $A_{s}$ is the saturation amplitude and $\tau$ is the averaging time. For the nanowires used in this work, the ultimate frequency stability at room temperature is of about $4 \times 10^{-8} \mathrm{~s}^{-1 / 2}$ that is a hundred-fold smaller than the experimental value. Therefore, an improvement of the frequency stability can be expected by optimizing the electrical noise, laser power stability and mechanical drift. A compelling feature of resonant nanowires is their capability for ultrasensitive mass sensing and for discerning subtle variations in the Young's modulus ${ }^{28}$, which point out these devices as promising tools for biological nanomechanical spectrometry ${ }^{11}$. In this sense, we have plotted the mass resolution based on the frequency stability values in figure $4 \mathrm{~b}$. Our current mass sensitivity is of $100 \mathrm{zg}$ for integration times of few seconds. The sensitivity can be largely enhanced by using smaller nanowires, higher vibration modes and by optimization of the readout set-up. The achievement of a mass resolution of $0.1 \mathrm{zg}\left(10^{-22} \mathrm{~g}\right)$ would open the door for weighing proteins with sensitivity down to a single aminoacid.

In conclusion, we demonstrate that the optical resonances associated to the light confinement in subwavelength semiconductor structures allow to efficiently extend optomechanics to nanoscale objects such as silicon nanowires. This discovery opens the door to 
novel cavity designs to efficiently cool tiny mechanical elements with the prospect of achieving the quantum limit more quickly than with micromechanical structures. On the other hand, this phenomenon enables the development of self-sustained nanowire oscillators in which the optical energy is converted into mechanical vibration at the natural frequency with unprecedented stability. These devices offer significant advantages with respect to previous frequency detection methods as there is no need of external excitation, which allows a high degree of simplification in the design of sensors based on nanowires. The concept of exploiting internal optical resonances together with the ever-increasing control in the fabrication of nanowires opens up immense opportunities to develop robust and reliable optonanomechanical devices driven by low light intensities.

\section{ASSOCIATED CONTENT}

Supporting Information. Fabrication details of the suspended silicon nanowires, description of the experimental set-up for measuring the displacement of the nanowires, and description of the theoretical model and numerical calculations. This material is available free of charge via the Internet at http:// pubs.acs.org.

\section{AUTHOR INFORMATION}

\section{Corresponding Author}

*E-mail: jtamayo@imm.cnm.csic.es.

\section{Author Contributions}

"These authors contributed equally.

\section{ACKNOWLEDGMENT}

The authors acknowledge financial support from the Spanish Science Ministry through the project TEC2009-14517-C02-01/02 and TEC2008-06756-C03-01. They also acknowledge Dr. Sheila Gónzalez by her technical support in the atomic force microscopy measurements of the distance between the nanowire and the substrate. 


\section{FIGURE CAPTIONS}

Figure 1. (a) Scanning electron microscopy image of a typical nanowire used in this work. The nanowires are normal to the trench wall where they were anchored with a length and diameter in the range of 6-8 $\mu \mathrm{m}$ and 100-200 $\mathrm{nm}$, respectively. (b) Cartoon of the light scattering pattern that emerges when a silicon nanowire coated with a $8 \mathrm{~nm}$ thick gold layer is illuminated by a laser beam. As a consequence of the gold coating, the fundamental vibration mode splits into two submodes (marked by the arrows) consisting on the nanowire vibrating parallel to the substrate (no detectable) and parallel to the optical axis at a higher frequency. (c) Theoretical calculation of the power absorption of the gold coated nanowires free in space as a function of the nanowire diameter. The series of peaks featuring the spectrum can be associated, to a very good approximation, with the Mie resonances. The images in the insets show the distributions of the square of the electric field module for the first four optical resonances labelled as $(0,0),(1,0),(2,0)$ and $(0,1)$. The image size is $300 \mathrm{~nm}$.

Figure 2. (a) Frequency spectra of the out of plane thermomechanical fluctuation of two nanowires referred to as NW 1 and NW 2 illuminated with a red $(633 \mathrm{~nm})$ and green $(543 \mathrm{~nm})$ laser. Both nanowires are separated from the substrate by 1450 $\mathrm{nm}$, however the diameters of NW 1 and NW 2 are 150 and $180 \mathrm{~nm}$, respectively. The modification in the cavity detuning by the wavelength switches the optomechanical amplification to cooling in NW 1 and vice versa in NW 2. The measurements were performed in a vacuum chamber at $10^{-7}$ Torr and room temperature. The laser beam spot size was of about $2 \mu \mathrm{m}$ and the incident power of $500 \mu \mathrm{W}$. (b) Amplitude at resonance of two nanowires as a function of the laser power intensity (symbols). The fundamental vibration mode of one nanowire is optically amplified (red symbols) whereas is cooled in the other case (cyan symbols). In the case of cooling, the achieved effective temperature is of about $50 \mathrm{~K}$ for a laser power of 500 $\mu \mathrm{W}$. In the case of optical amplification, the amplitude moderately grows with the laser intensity until it undergoes an abrupt increase of about 20 times when the laser power is $310 \mu \mathrm{W}$. Above this critical laser power, the nanowire exhibits selfsustained oscillation. The blue dashed lines represent the fitting to the theory (see eq. (1) in the related text). (c) Schematic of the feedback loop that describes the optomechanical coupling. The thermomechanical fluctuations modify the nanowire position with respect to the optical field that approximately consists of a standing wave due to the interference between the incoming radiation and the reflected beam off the substrate. The mechanical fluctuations then change the photothermal force exerted by the optical field on the nanowire. Thereby, the mechanical resonator is self-driven by a force that is proportional to its motion with a phase lag that accounts for heat diffusion time.

Figure 3. (a) Calculated color intensity map of the optomechanical feedback gain of the cavity per light power unit for at wavelength of $633 \mathrm{~nm}$, respectively, as a function of the distance between the nanowire and the substrate and the diameter of the nanowire, both normalized to the wavelength. The calculations were performed for an incidence power of $500 \mu \mathrm{W}$ and a beam spot size of $2 \mu \mathrm{m}$. The contour lines separate the regions of cooling and amplification. The experimental diameter and cavity distance coordinates of the nanowires in which amplification or cooling was observed are included as red and cyan circles, showing a good agreement with the theoretical predictions. (b) Power absorption of the gold coated nanowires vs the nanowire diameter when they are situated at 1250 and $1400 \mathrm{~nm}$ from the substrate, respectively. The interference field modulates the power absorbed by the electromagnetic confined modes within the nanowire. This effect is particularly significant in the absorption of the modes $(1,0)$ and $(2,0)$ (c) Electrical intensity distribution around the nanowires for the modes $(1,0)$ and $(2,0)$ at 1250 and $1400 \mathrm{~nm}$ from the substrate (image size is $1500 \mathrm{~nm}$ ). The nanowire and the substrate are outlined as a circle and a line at the bottom of each image, respectively. The color bar intensity shows the relative electrical intensity.

Figure 4. (a) One hour recording of the relative frequency deviations of the self-sustained nanowire oscillator. The integration time is of one second and the frequency is of $3505 \mathrm{kHz}$. The red dashed lines represent the standard deviation of the frequency. (b) Standard deviation of the relative frequency shifts and corresponding mass resolution as a function of the integration time. The mass resolution is approximately given by the product of two times the effective mass of the nanowires 
$(\approx 50 \mathrm{fg})$. The red dashed line represents the ultimate frequency stability imposed by the thermomechanical limit at room temperature.

\section{REFERENCES}

1. Martin, Y.; Williams, C.; Wickramasinghe, H. K. Journal of applied physics 1987, 61, (10), 4723-4729.

2. $\quad$ Thundat, T.; Wachter, E.; Sharp, S.; Warmack, R. Applied Physics Letters 1995, 66, 1695.

3. $\quad$ Fanget, S.; Hentz, S.; Puget, P.; Arcamone, J.; Matheron, M.; Colinet, E.; Andreucci, P.; Duraffourg, L.; Meyers, E.; Roukes, M. Sensors and Actuators B: Chemical 2011.

4. Waggoner, P. S.; Craighead, H. G. Lab on a Chip 2007, 7, (10), 1238-1255.

5. $\quad$ Arlett, J.; Myers, E.; Roukes, M. Nature Nanotechnology 2011, 6, (4), 203-

215.

6. Ekinci, K.; Yang, Y.; Roukes, M. Journal of applied physics 2004, 95, 2682.

7. Yang, Y.; Callegari, C.; Feng, X.; Ekinci, K.; Roukes, M. Nano letters 2006, 6, (4), 583-586.

8. Jensen, K.; Kim, K.; Zettl, A. Nature Nanotechnology 2008, 3, (9), 533-537.

9. $\quad$ Feng, X.; He, R.; Yang, P.; Roukes, M. Nano letters 2007, 7, (7), 1953-1959.

10. Lassagne, B.; Garcia-Sanchez, D.; Aguasca, A.; Bachtold, A. Nano letters 2008, 8, (11), 3735-3738.

11. Naik, A.; Hanay, M.; Hiebert, W.; Feng, X.; Roukes, M. Nature Nanotechnology 2009, 4, (7), 445-450.

12. Zande, A. M.; Barton, R. A.; Alden, J. S.; Ruiz-Vargas, C. S.; Whitney, W. S.; Pham, P. H. Q.; Park, J.; Parpia, J. M.; Craighead, H. G.; McEuen, P. L. Nano letters 2010.

13. Degen, C.; Poggio, M.; Mamin, H.; Rettner, C.; Rugar, D. Proceedings of the National Academy of Sciences 2009, 106, (5), 1313.

14. Li, M.; Tang, H.; Roukes, M. Nature Nanotechnology 2007, 2, (2), 114-120.

15. Kippenberg, T. J.; Vahala, K. J. Science 2008, 321, (5893), 1172.

16. Favero, I.; Karrai, K. Nature Photonics 2009, 3, (4), 201-205.

17. Marquardt, F.; Girvin, S. Physics 2009, 2, 40.

18. Brennecke, F.; Ritter, S.; Donner, T.; Esslinger, T. Science 2008, 322, (5899), 235.

19. Anetsberger, G.; Arcizet, O.; Unterreithmeier, Q.; Rivière, R.; Schliesser, A.; Weig, E.; Kotthaus, J.; Kippenberg, T. J. Nature Physics 2009, 5, (12), 909-914.

20. Favero, I.; Metzger, C.; Camerer, S.; Konig, D.; Lorenz, H.; Kotthaus, J.;

Karrai, K. Applied Physics Letters 2007, 90, (10), 104101-104101-3.

21. Gröblacher, S.; Hertzberg, J. B.; Vanner, M. R.; Cole, G. D.; Gigan, S.; Schwab, K.; Aspelmeyer, M. Nature Physics 2009, 5, (7), 485-488.

22. Van Thourhout, D.; Roels, J. Nature Photonics 2010, 4, (4), 211-217.

23. Ding, L.; Baker, C.; Senellart, P.; Lemaitre, A.; Ducci, S.; Leo, G.; Favero, I. Physical review letters 2010, 105, (26), 263903.

24. Thompson, J.; Zwickl, B.; Jayich, A.; Marquardt, F.; Girvin, S.; Harris, J. Nature 2008, 452, (7183), 72-75.

25. Chan, J.; Alegre, T. P. M.; Safavi-Naeini, A. H.; Hill, J. T.; Krause, A.; Groblacher, S.; Aspelmeyer, M.; Painter, O. Nature 2011, 478, (7367), 89-92. 
26. O'Connell, A.; Hofheinz, M.; Ansmann, M.; Bialczak, R. C.; Lenander, M.; Lucero, E.; Neeley, M.; Sank, D.; Wang, H.; Weides, M. Nature 2010, 464, (7289), 697-703.

27. Teufel, J.; Li, D.; Allman, M.; Cicak, K.; Sirois, A.; Whittaker, J.;

Simmonds, R. Nature 2011, 471, (7337), 204-208.

28. Gil-Santos, E.; Ramos, D.; Martínez, J.; Fernández-Regúlez, M.; García, R.; San Paulo, Á.; Calleja, M.; Tamayo, J. Nature Nanotechnology 2010, 5, (9), 641645.

29. Nichol, J. M.; Hemesath, E. R.; Lauhon, L. J.; Budakian, R. Applied Physics Letters 2008, 93, (19), 193110-193110-3.

30. Biedermann, L. B.; Tung, R. C.; Raman, A.; Reifenberger, R. G. Nanotechnology 2009, 20, 035702.

31. Karabacak, D.; Kouh, T.; Ekinci, K. Journal of applied physics 2005, 98, 124309.

32. Pini, V.; Tamayo, J.; Gil-Santos, E.; Ramos, D.; Kosaka, P.; Tong, H. D.; van Rijn, C.; Calleja, M. ACS nano.

33. Ramos, D.; Tamayo, J.; Mertens, J.; Calleja, M. Journal of applied physics 2006, 99, 124904.

34. Cao, L.; Fan, P.; Vasudev, A. P.; White, J. S.; Yu, Z.; Cai, W.; Schuller, J. A.; Fan, S.; Brongersma, M. L. Nano letters 2010, 10, (2), 439-445.

35. Cao, L.; White, J. S.; Park, J. S.; Schuller, J. A.; Clemens, B. M.;

Brongersma, M. L. Nature Materials 2009, 8, (8), 643-647.

36. Tamayo, J.; Calleja, M.; Ramos, D.; Mertens, J. Physical Review B 2007, 76, (18), 180201.

37. Feng, X.; White, C.; Hajimiri, A.; Roukes, M. Nature Nanotechnology 2008, 3, (6), 342-346.

38. Ham, D.; Hajimiri, A. Solid-State Circuits, IEEE Journal of 2003, 38, (3), 407-418. 
Figure 1
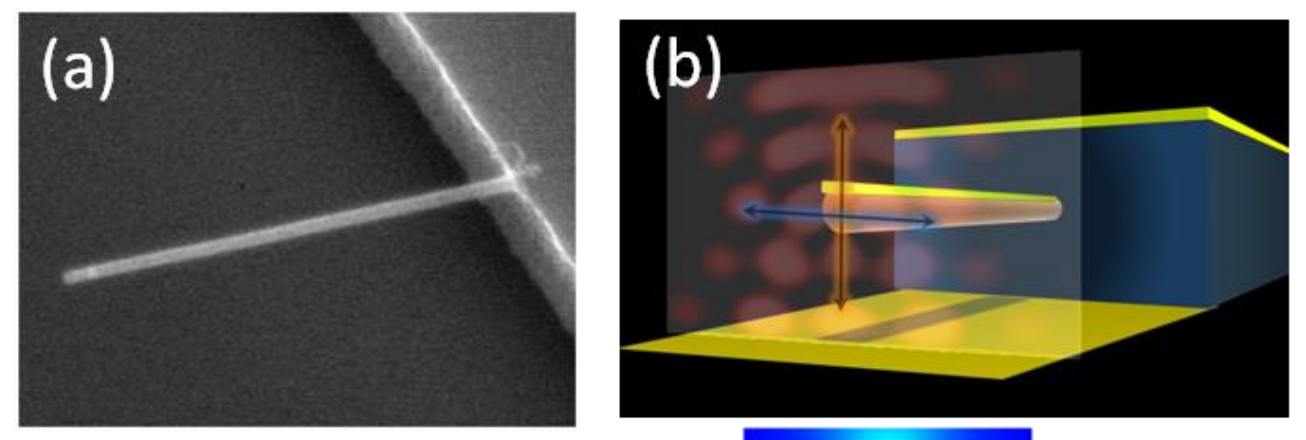

(c)

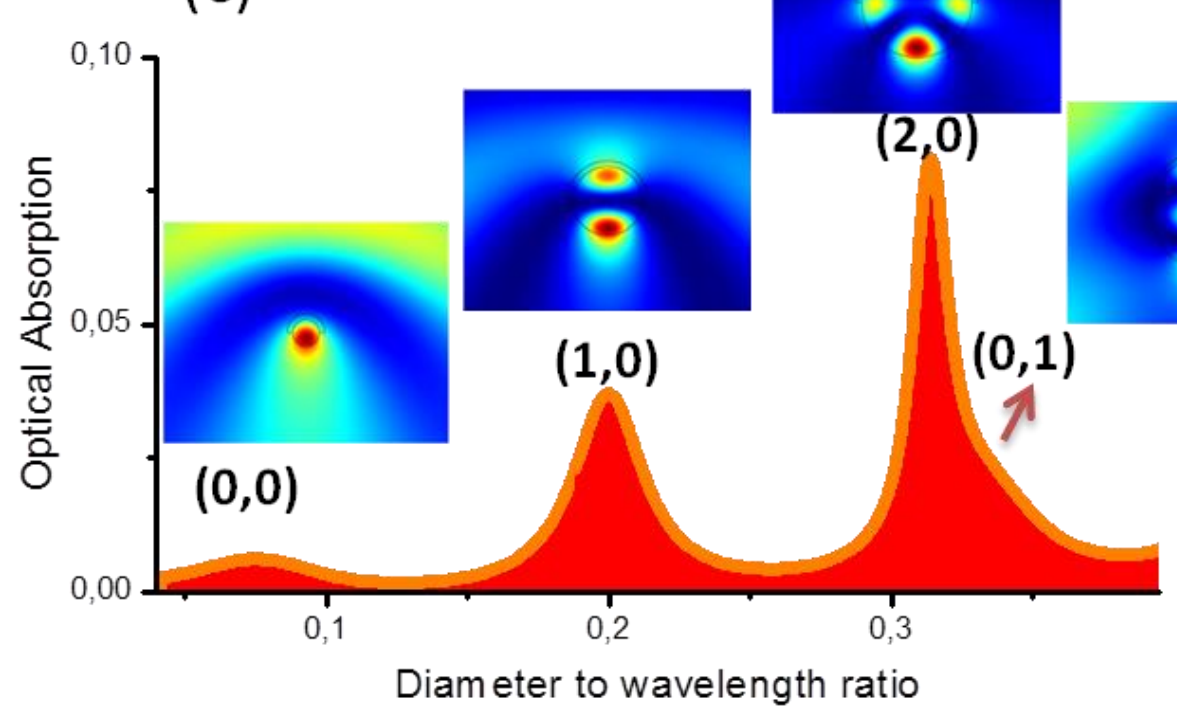




\section{Figure 2}
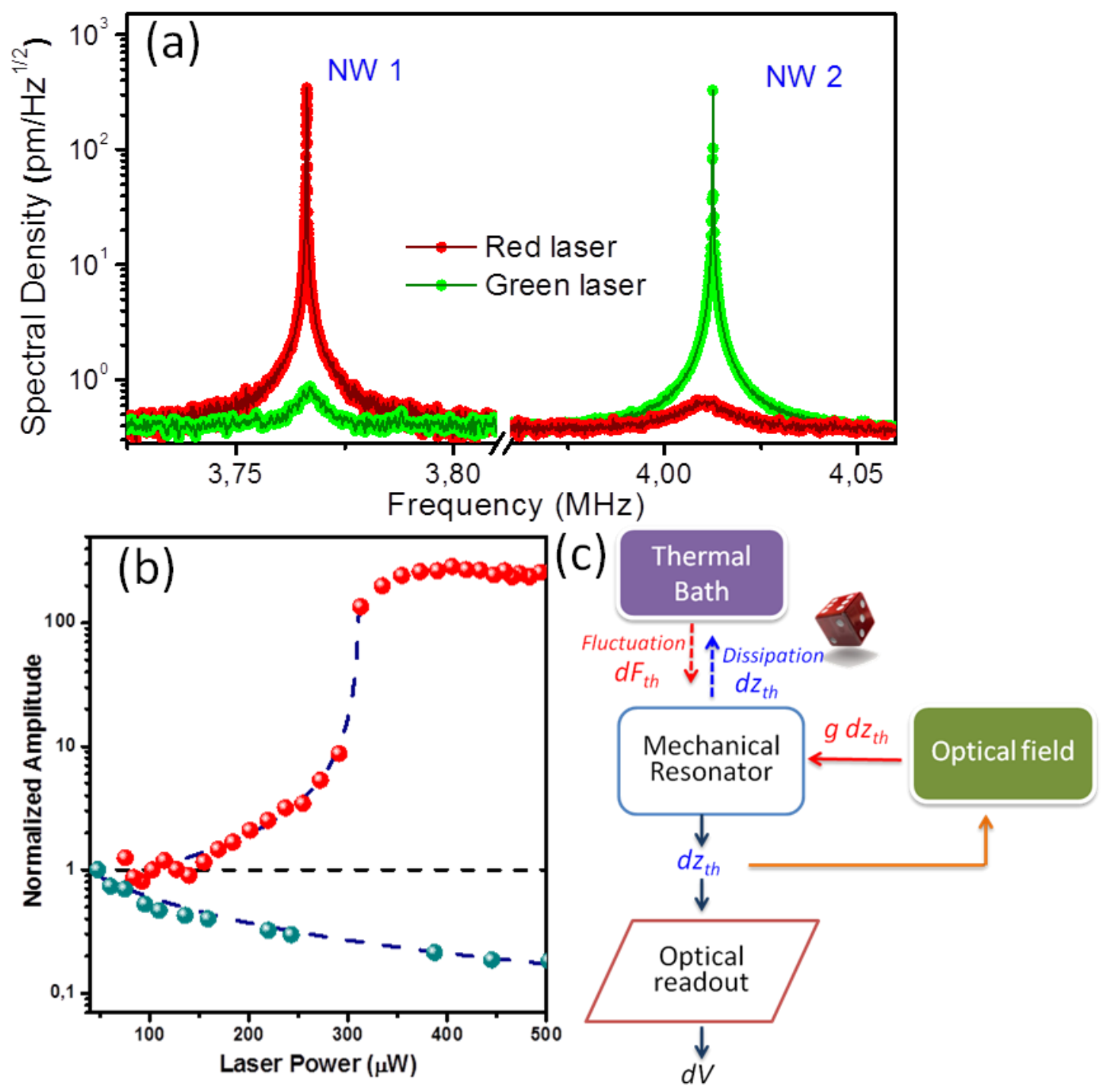


\section{Figure 3}

(a)

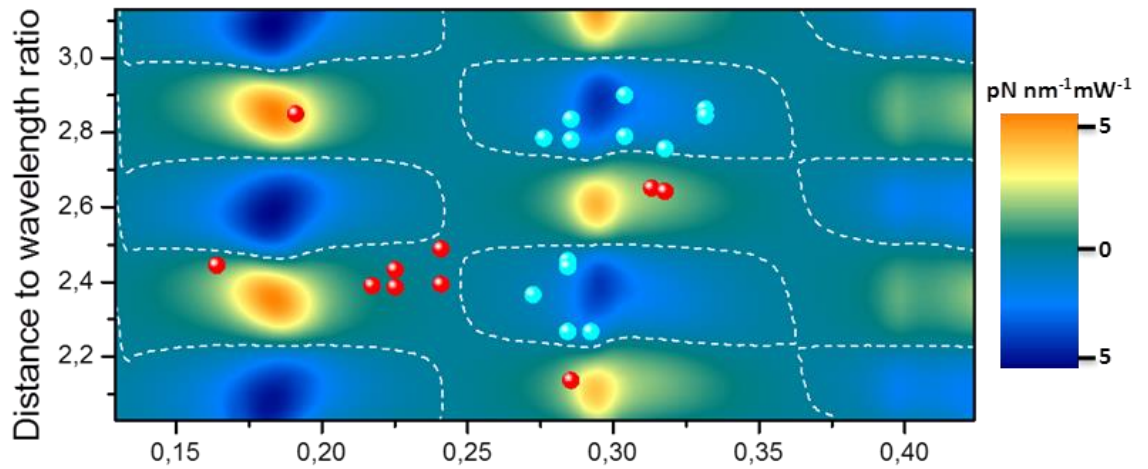

(b)

Diameter to wavelength ratio

(c)

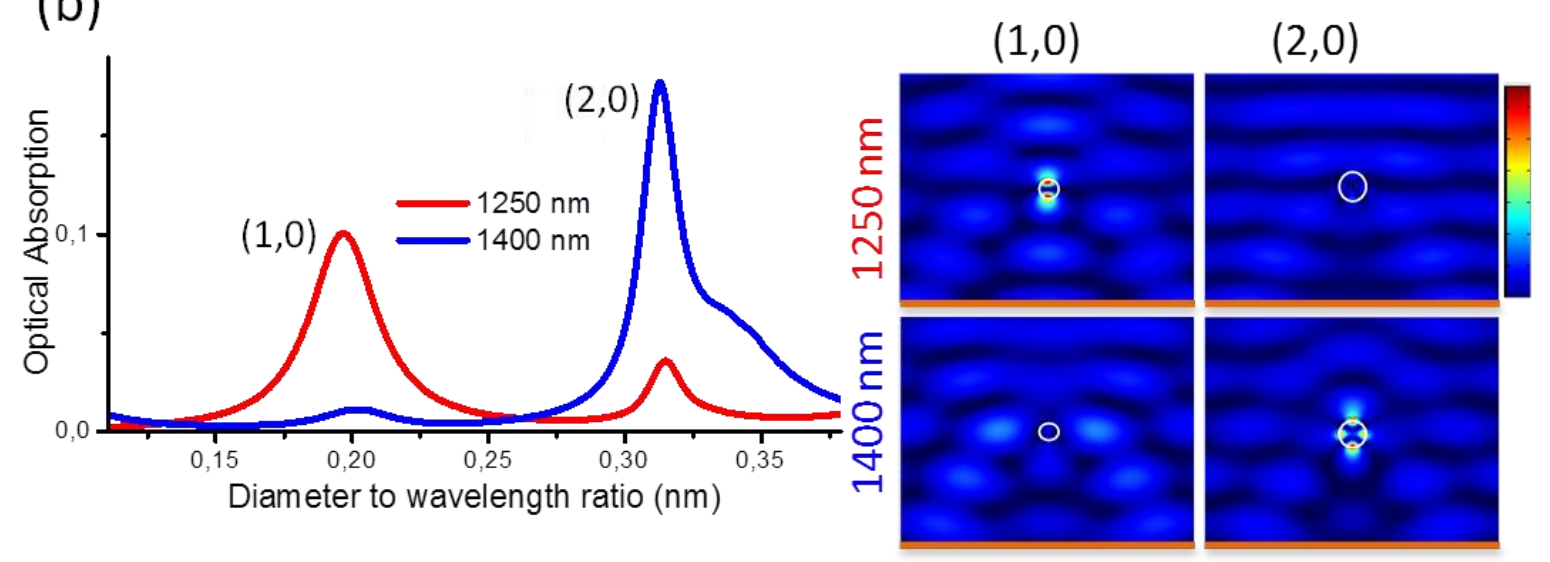




\section{Figure 4}

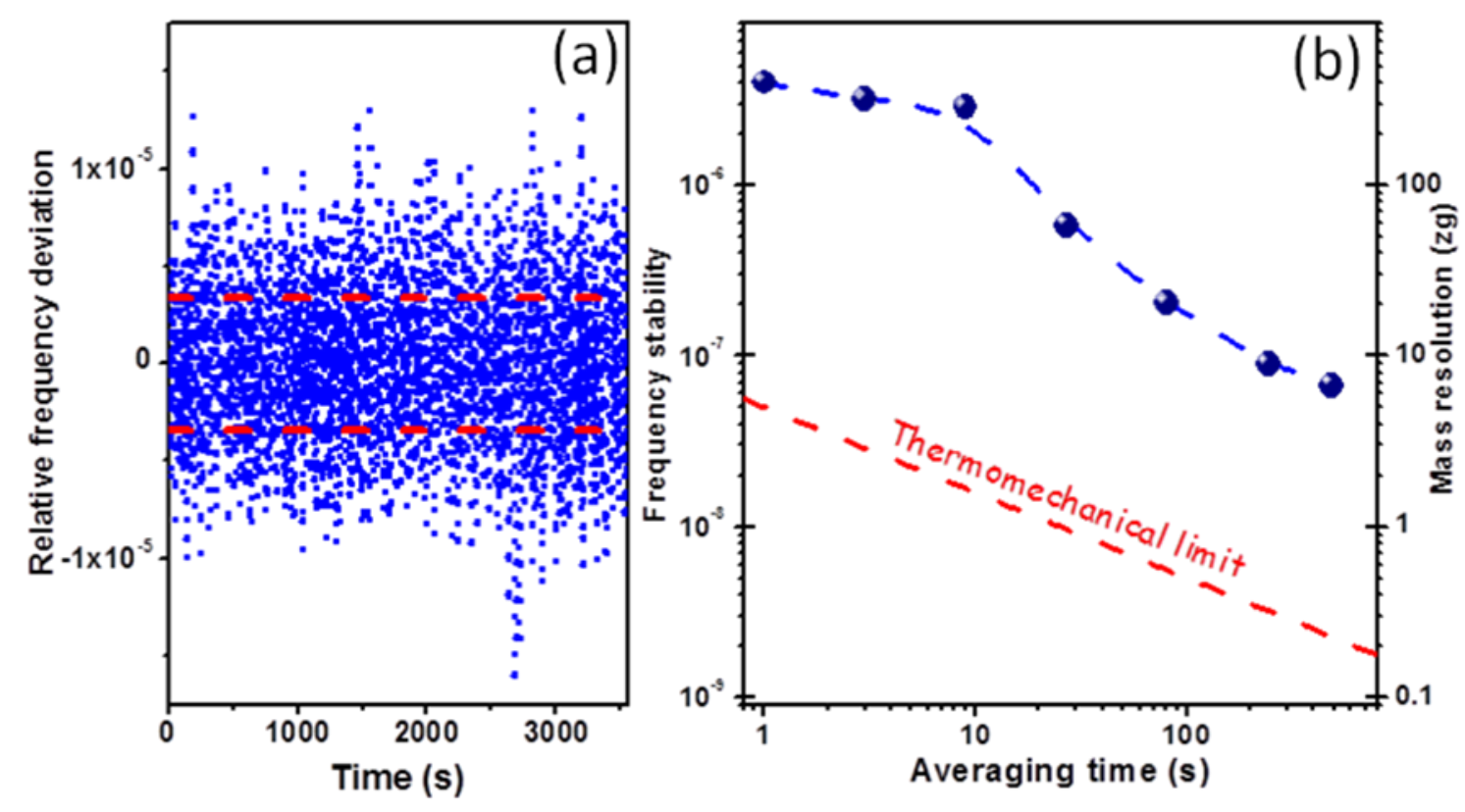

